\title{
Upregulation of Isoflavonoids and Soluble Proteins in Edible Legumes by Light and Fungal Elicitor Treatments
}

\author{
ASHISH LAL, M.Sc., ${ }^{1}$ SARA WARBER, M.D., ${ }^{2}$ ARA KIRAKOSYAN, Ph.D., ${ }^{1,2}$ \\ PETER B. KAUFMAN, Ph.D., ${ }^{1,2}$ and JAMES A. DUKE, Ph.D. ${ }^{3}$
}

\begin{abstract}
Objective: In this study, our working hypothesis was that continuous light and fungal elicitation treatment of legume seedlings would lead to enhanced levels of isoflavonoids and soluble proteins.

Results: Based on short-term light and dark treatments, isoflavonoid (genistein, genistin, daidzein, and daidzin) and soluble protein concentrations were significantly upregulated in the "light" environment compared to the "dark" environment for all edible legume species (kudzu vine, soybean, garbanzo bean, fava bean, mung bean, adzuki bean) that were tested. Kudzu seedlings showed the highest levels of both isoflavonoids and soluble proteins after light-elicited upregulation compared to the other legumes analyzed. All legumes showed less up-regulation of isoflavonoid synthesis when treated with Phytophtora sojae fungal elicitor. Oligosaccharide fungal elicitor caused no such upregulation.

Conclusions: The findings in this study show that edible legume seedlings have enhanced levels of isoflavonoids and soluble proteins when they are grown in the light compared to the conventional practice of growing such seedlings in the dark. This will clearly result in significant improvement in their nutritive and medicinal value.
\end{abstract}

\section{INTRODUCTION}

$\mathbf{M}$ any legumes in the bean family (Fabaceae) are important sources of isoflavones and soluble dietary protein. Our interest in these compounds derives from the fact that isoflavonoids such as genistein and daidzein are important medicinal compounds (reviewed by Boik, 1996; Duke, 1995). Genistein is a promising anticancer agent that inhibits platelet aggregation, induces apoptosis, inhibits leukotriene production, inhibits DNA topoisomerase II, inhibits angiogenesis, reduces the bioavail- ability of sex hormones, and induces differentiation in cancer cells (Boik, 1996; Fotsis et al., 1995). Genistein and daidzein both have phytoestrogen activity. Because of their estrogenic activity, these isoflavones are important in the treatment of estrogen-dependent cancers (Kaufman et al., 1997). Daidzein, as well as daidzin, has been shown to inhibit the enzyme, aldehyde dehydrogenase (ALDH-I), and nicotinamideadenine dinucleotide (NAD)-dependent ALDH that calayzes the oxidation of acetaldehyde, the primary product of alcohol metabolism (Duke, 1995; Keung and Vallee, 1993a,

\footnotetext{
${ }^{1}$ Department of Molecular, Cellular, and Developmental Biology, University of Michigan, Ann Arbor, MI.

${ }^{2}$ Complementary and Alternative Medicine Research Center, University of Michigan, Ann Arbor, MI.

${ }^{3}$ Herbal Vineyard, Inc., Fulton, MD.
} 
1993b). As such, it has been investigated as a treatment for alcoholism.

In the present investigation, we hypothesized that seedlings of selected edible legumes, when provided with continuous illumination, would show increased levels of genistein and daidzein and their glucosyl conjugates, as well as soluble proteins, compared to seedlings grown in the dark. This hypothesis is based on the supposition that light-mediated photosynthetic fixation of carbon dioxide $\left(\mathrm{CO}_{2}\right)$ will yield higher levels of isoflavonoids and soluble proteins. Corollary to this hypothesis, we postulated that treatment with fungal (Phytophthora soji) cell wall preparations, as well as cell wall oligosaccharides, would have similar upregulating effects. The basis for this reasoning is the fact that when legumes such as soybean (Glycine max) are attacked by a fungal pathogen such as Phytophthora soji, the legumes increase their synthesis of isoflavonoids through transcriptional upregulation caused by fungal cell wall polysaccharide molecules. In turn, the enhanced levels of genistein in the host plant kill or retard growth of the fungus, and this is the basis for disease resistance in the plant (Dixon et al., 1983).

The primary objective of the present investigation was to determine whether or not continuous exposure to light or transient fungal elicitor treatments could elicit increases in the levels of genistein and daidzein and their glucosyl conjugates as well as soluble proteins in germinating seedlings of selected edible legumes. We show that such treatments do have a positive upregulating effect on synthesis of these isoflavonoids and of soluble proteins in all of the legume seedlings tested, but to the greatest extent, in kudzu seedlings (Pueraria montana). Of the two comparisons, light has the greatest upregulating effect. These results have important implications for human nutrition and complementary and alternative medicine.

\section{MATERIALS AND METHODS}

\section{Seed sources}

Seeds of Japanese Kudzu (Pueraria montana) were obtained from Adams-Briscoe Seed Company, Inc., (Jackson, GA). The seeds from this source are treated with a red-colored fungicide to protect the seedlings from damping-off disease. We strongly advise that seedlings derived from such seeds not be used for human consumption. Seeds of all other legumes tested were obtained from Johnny's Selected Seeds, (Albion, ME); these seeds were not treated with fungicide.

\section{Photoperiod manipulations}

Legumes for all experiments were germinated and grown in Bio Set ${ }^{\mathrm{TM}}$ seed sprouting units (Fig. 1). Bio Set units were obtained from Johnny's Selected Seeds. Control and experimental environments were simulated in photobioreactors using Precision Scientific Co. Dual Program Illuminated Incubators (Chicago, IL; model no. 818).

To test the effects of light on the synthesis of isoflavonoids and soluble protein in the various legumes, the incubators were programmed in the following manner: control, 16 hours of light and 8 hours of darkness (with 1 hour of light interruption in the middle of the dark period); light, 24 hours of light; dark, 24 hours of darkness. The temperature of the incubators was maintained at $30^{\circ} \mathrm{C}$. Light intensity on the Bio Set units was $162 \mu \mathrm{E} \cdot \mathrm{m}^{-2} \cdot \mathrm{s}^{-1}$. Seedlings in the Bio Set units were watered twice daily. On emergence of the first true leaves, whole seedlings were harvested, washed with distilled water, and ground into a fine powder using liquid nitrogen. This powder was immediately stored at $-80^{\circ} \mathrm{C}$ prior to extraction and analysis.

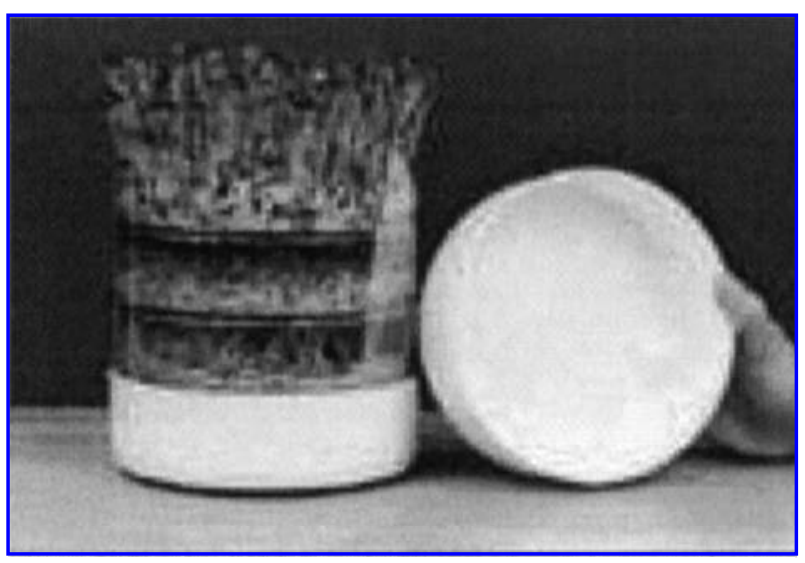

FIG. 1. A Bio Set ${ }^{\mathrm{TM}}$ (Johnny's Selected Seeds, Albion, $\mathrm{ME)}$ seed sprouting unit. 


\section{Fungal elicitor treatments}

To test the effects of fungal elicitor on the synthesis of isoflavonoids and soluble protein in the legumes, all legumes were grown in an incubator programmed for 16 hours of light and 8 hours of darkness (with 1 hour of light interruption). Temperature was maintained at $30^{\circ} \mathrm{C}$. Light intensity was $162 \mu \mathrm{E} \cdot \mathrm{m}^{-2} \cdot \mathrm{s}^{-1}$. Seedlings were watered twice daily. On the emergence of the first true leaves, seedlings were harvested from the Bio Sets and submerged for 30 minutes in either Phytophthora sojae or oligosaccharide standard solutions provided by Michael Hahn, B.Sc., M.Sc., Ph.D., Complex Carbohydrate Research Center, USDA, Athens, GA), or distilled water (control). These seedlings were transplanted back to the Bio Sets for continued growth for 4 more days.

After 4 days, whole seedlings for each treatment were harvested, washed with distilled water, and ground into a fine powder using liquid nitrogen. The powder was immediately stored at $-80^{\circ} \mathrm{C}$ prior to extraction, high performance liquid chromatography (HPLC) analysis of isoflavonoids, and spectrophotometric analysis of soluble proteins.

\section{Isoflavonoid extraction}

Extraction was carried out as described by Kaufman et al. (Kaufman et al., 1997, 2002). Triplicate samples, $0.5 \mathrm{~g}$ each, of the fine powder from each experiment were prepared, two of which were placed in two test tubes containing $4 \mathrm{~mL}$ of $80 \% \mathrm{MeOH}$. These samples were then vortexed and the supernatant was collected and placed in $15 \mathrm{~mL}$ Corex $^{\mathrm{TM}}$ centrifuge tubes. This was repeated three times for each test tube. Tubes were then centrifuged for 20 minutes at $27,000 \mathrm{~g}$ at $22^{\circ} \mathrm{C}$ in a DuPont Sorvall RC-5 Superspeed refrigerated centrifuge (DuPont Instruments, Newtown, CT). The supernatant was then diluted to a $30 \% \mathrm{MeOH}$ solution and then run through a Waters SepPak $^{\mathrm{TM}}$ Vac C-18 cartridge chromatography column (Waters Corp., Milford, MA). These cartridges were placed on a faucet-type vacuum aspirator apparatus that gently drew the supernatant through the Sep-Pak cartridges. Prior to running extracts through the C-18 cartridges, the cartridges were pre-equilibrated by wash- ing them with $\mathrm{MeOH}$, first $100 \%$, then $80 \%$, and finally $30 \%$. The samples were then eluted from the cartridges using $100 \% \mathrm{MeOH}$. The samples containing the isoflavonoids were then airdried. Next, $1 \mathrm{~mL}$ of $80 \% \mathrm{MeOH}$ was added to the air-dried test tubes. The tubes were then vortexed, covered with Parafilm, ${ }^{\mathrm{TM}}$ (Pechiney Plastic Packaging Inc., Neenah, WI) and kept in a refrigerator at $4^{\circ} \mathrm{C}$ until HPLC analysis.

\section{HPLC analysis}

Fifteen microliters $(15 \mu \mathrm{L})$ of each sample were assayed by reverse phase (ODS) HPLC (using a Vydac C18 column, $4.6 \times 250 \mathrm{~mm}$ in size) at $280-\mathrm{nm}$ wavelength using a linear gradient from $\mathrm{AcCN}: \mathrm{H}_{2} \mathrm{O}$ :TFA (20:79.9:0.1) to $\mathrm{MeCN}: \mathrm{H}_{2} \mathrm{O}$ :TFA (86:13.9:0.1). Genistein, genistin, daidzein, and daidzin concentrations were determined using standard samples of each. These isoflavonoid standards were obtained from Sigma-Aldrich (St. Louis, MO). These methods are cited in Kaufman et al. (Kaufman et al., 1997).

\section{Protein assay}

The soluble proteins were extracted from homogenized sprouts in $80 \%$ methanol. After filtration through a $0.45-\mu \mathrm{m}$ filter, the extracts were ready for soluble protein assay. The protein assay method of Bradford (1976) was used. It is based on the proportional binding of the dye, Coomassie blue, to soluble proteins. Within the linear range of the assay (approximately 5 to $25 \mathrm{mg} \cdot \mathrm{mL}^{-1}$ ), the more protein present, the more Coomassie dye binds. The protein concentrations of the experimental samples were determined by comparison to a known protein standard: bovine serum albumin (BSA) $1 \mathrm{mg} \cdot \mathrm{ml}^{-1}$. Absorbance of soluble proteins were assayed quantitatively at $595 \mathrm{~nm}$ with a Shimadzu UV160U UV-Visible recording spectrophotometer (Shimadzu Scientific Instruments, Columbia, MD).

\section{Statistical analysis of data}

Experiments were repeated at least three times, and the data were analyzed statistically. All results are given as mean \pm standard deviation (SD). Differences between variables were 
tested for significance by Student's $t$ test. A $p$ value of $<0.05$ was considered to be significant.

\section{RESULTS}

Seedlings of six different taxa of edible legumes were subjected to an alteration of environmental and molecular factors in an attempt to upregulate isoflavonoids, their glycosyl conjugates, and soluble proteins. Analyses were conducted on entire seedlings. Of the samples studied, each showed significant increases of the aforementioned compounds following treatments (equal to or greater than a $30 \%$ increase) (Tables 1-6). Of special interest are Pueraria montana (kudzu) (Table 1A and 1B) and Cicer arietinum (garbanzo bean) (Table 3A and 3B). P. montana showed the greatest isoflavonoid increases of $80 \%$ and $47 \%$ after a change in the environmental and biotic parameters, respectively, while C. arietinum demon- strated the highest increases of soluble proteins, namely, $63 \%$ and $90 \%$, respectively.

While the fungal elicitor, $P$. sojae, prompted significant increases (of at least 25\%) for both isoflavonoids, their glycosyl derivatives and soluble proteins in all species treated, those seedlings treated with oligosaccharide solution show no significant change in concentrations of either soluble proteins or isoflavonoids (Tables $1 \mathrm{~B}-6 \mathrm{~B})$. It is possible that the oligosaccharide molecules were not absorbed by the legumes during the treatment period.

The data in Tables 1-6 clearly show that light exposure increases the concentrations of both isoflavonoids and soluble proteins compared to dark control values. Seedlings left in the dark environment show significant decreases in isoflavonoid and soluble protein levels (averaging $-23 \%$ and $-32 \%$, respectively). These decreases are most likely caused by the absence of photosynthetic carbon-fixation in the dark environment. This is the key process in green

Table 1A. Isoflavonoid Concentrations $\left(\mathrm{mg} \cdot \mathrm{kg}^{-1} \text { Dry Weight Biomass) }\right)^{\mathrm{a}}$ In Kudzu (Pleraria montana)

\begin{tabular}{|c|c|c|c|c|c|c|}
\hline \multirow[b]{2}{*}{ Isoflavonoids } & \multicolumn{3}{|c|}{ Environmental factors } & \multicolumn{3}{|c|}{ Biotic factors (fungal elicitor) } \\
\hline & Control & Light & Dark & Control & P. sojae & Oligosaccharide \\
\hline \multirow[t]{2}{*}{ Daidzein } & 906.5 & $1,350.3$ & 800.1 & 750.0 & 955.3 & 744.2 \\
\hline & \pm 80.2 & \pm 132.5 & \pm 45.2 & \pm 25.2 & \pm 75.9 & \pm 88.1 \\
\hline \multirow[t]{2}{*}{ Genistein } & 256.1 & 432.1 & 184.3 & 250.6 & 302.3 & 275.3 \\
\hline & \pm 21.5 & \pm 25.2 & \pm 7.5 & \pm 14.0 & \pm 25.0 & \pm 35.2 \\
\hline \multirow[t]{2}{*}{ Genistin } & 140.0 & 655.0 & 0 & 175.3 & 503.5 & 180.5 \\
\hline & \pm 13.3 & \pm 80.5 & & \pm 15.1 & \pm 11.9 & \pm 10.0 \\
\hline \multirow[t]{2}{*}{ Daidzin } & 222.9 & 302.5 & 145.3 & 211.3 & 274.3 & 224.1 \\
\hline & \pm 15.9 & \pm 20.9 & \pm 8.5 & \pm 25.3 & \pm 38.5 & \pm 11.4 \\
\hline \multirow[t]{2}{*}{ Total } & 1525.5 & 2739.9 & 1129.7 & 1387.2 & 2035.4 & 1424.1 \\
\hline & \pm 130.9 & \pm 259.1 & \pm 101.2 & \pm 129.6 & \pm 151.3 & \pm 144.7 \\
\hline $\begin{array}{l}\text { Percent of } \\
\text { control (\%) }\end{array}$ & 100 & 180 & 74 & 100 & 147 & 103 \\
\hline
\end{tabular}

aThree independent experiments, $n=3$; values are means \pm standard deviation (SD).

Table 1B. Soluble Protein Concentrations $\left(\mathrm{mg} \cdot \mathrm{kg}^{-1} \text { Dry weight biomass) }\right)^{\mathrm{a}}$ in Kudzu (Pueraria montana)

\begin{tabular}{|c|c|c|c|c|c|}
\hline \multicolumn{3}{|c|}{ Environmental factors } & \multicolumn{3}{|c|}{ Biotic factors (fungal elicitor) } \\
\hline Control & Light & Dark & Control & P. sojae & Oligosaccharide \\
\hline $\begin{array}{r}31,007.3 \\
\pm 2536.3\end{array}$ & $\begin{array}{r}40,125.0 \\
\pm \quad 2805.1\end{array}$ & $\begin{array}{r}25,055.3 \\
+\quad 1555.0\end{array}$ & $\begin{array}{r}28,055.0 \\
+20006\end{array}$ & $\begin{array}{r}35,155.5 \\
+\quad 20571\end{array}$ & $\begin{array}{r}20,577.8 \\
+\quad 18531\end{array}$ \\
\hline Control (\%) & $\begin{array}{l}\text { Percent of } \\
\quad \text { control }(\%)\end{array}$ & $\begin{array}{l}\text { Percent of } \\
\text { control }(\%)\end{array}$ & Control (\%) & $\begin{array}{l}\text { Percent of } \\
\text { control (\%) }\end{array}$ & $\begin{array}{l}\text { Percent of } \\
\text { control }(\%)\end{array}$ \\
\hline 100 & 130 & 81 & 100 & 125 & \\
\hline
\end{tabular}

aThree independent experiments, $n=3$; values are means \pm standard deviation (SD). 
Table 2A. Isoflavonoid Concentrations $\left(\mathrm{mg} \cdot \mathrm{kg}^{-1}\right.$ Dry Weight Biomass) ${ }^{\mathrm{a}}$ in Soybean (GLyCine MaX)

\begin{tabular}{|c|c|c|c|c|c|c|}
\hline \multirow[b]{2}{*}{ Isoflavonoids } & \multicolumn{3}{|c|}{ Environmental factors } & \multicolumn{3}{|c|}{ Biotic factors (fungal elicitor) } \\
\hline & Control & Light & Dark & Control & P. sojae & Oligosaccharide \\
\hline \multirow[t]{2}{*}{ Daidzein } & 45.3 & 55.8 & 32.3 & 40.1 & 48.2 & 38.1 \\
\hline & \pm 3.2 & \pm 4.4 & \pm 2.2 & \pm 4.5 & \pm 4.1 & \pm 3.8 \\
\hline \multirow[t]{2}{*}{ Genistein } & 55.3 & 68.2 & 48.6 & 44.9 & 49.1 & 42.6 \\
\hline & \pm 4.1 & \pm 5.9 & \pm 3.9 & \pm 3.3 & \pm 8.2 & \pm 4.1 \\
\hline \multirow[t]{2}{*}{ Genistin } & 22.1 & 31.6 & 17.2 & 15.0 & 19.6 & 16.2 \\
\hline & \pm 1.1 & \pm 2.2 & \pm 2.7 & \pm 0.7 & \pm 5.1 & \pm 1.9 \\
\hline \multirow[t]{2}{*}{ Daidzin } & 11.5 & 15.3 & 6.5 & 8.0 & 13.3 & 7.6 \\
\hline & \pm 0.7 & \pm 0.9 & \pm 0.4 & \pm 0.3 & \pm 0.9 & \pm 0.5 \\
\hline \multirow[t]{2}{*}{ Total } & 134.2 & 170.9 & 104.6 & 108.0 & 130.2 & 104.5 \\
\hline & \pm 9.1 & \pm 13.4 & \pm 9.2 & \pm 8.8 & \pm 18.3 & \pm 10.3 \\
\hline $\begin{array}{l}\text { Percent of } \\
\text { control }(\%)\end{array}$ & 100 & 128 & 78 & 100 & 120 & 96 \\
\hline
\end{tabular}

aThree independent experiments, $n=3$; values are means \pm standard deviation (SD).

Table 2B. Soluble Protein Concentrations $\left(\mathrm{mg} \cdot \mathrm{kg}^{-1} \text { Dry Weight biomass) }\right)^{\mathrm{a}}$ in Soybean (Glycine max)

\begin{tabular}{|c|c|c|c|c|c|}
\hline \multicolumn{3}{|c|}{ Environmental factors } & \multicolumn{3}{|c|}{ Biotic factors (fungal elicitor) } \\
\hline Control & Light & Dark & Control & P. sojae & Oligosaccharide \\
\hline $\begin{array}{l}18,055.3 \\
\pm 912.3\end{array}$ & $\begin{array}{r}22,200.3 \\
\pm 1001.5\end{array}$ & $\begin{array}{l}15,509.0 \\
\pm 798.6\end{array}$ & $\begin{array}{r}16,838.3 \\
\pm 1205.7\end{array}$ & $\begin{array}{r}21,555.0 \\
\pm 1855.5\end{array}$ & $\begin{array}{r}17,000.0 \\
\pm 1504.3\end{array}$ \\
\hline Control (\%) & $\begin{array}{l}\text { Percent of } \\
\text { control }(\%)\end{array}$ & $\begin{array}{l}\text { Percent of } \\
\quad \text { control }(\%)\end{array}$ & Control (\%) & $\begin{array}{l}\text { Percent of } \\
\quad \text { control }(\%)\end{array}$ & $\begin{array}{l}\text { Percent of } \\
\quad \text { control }(\%)\end{array}$ \\
\hline 100 & 123 & 86 & 100 & 128 & 101 \\
\hline
\end{tabular}

aThree independent experiments, $n=3$; values are means \pm standard deviation (SD).

Table 3A. Isoflavonoid Concentrations $\left(\mathrm{mg} \cdot \mathrm{kg}^{-1}\right.$ Dry weight biomass) ${ }^{\mathrm{a}}$ in Garbanzo Bean (Cicer arietinum)

\begin{tabular}{|c|c|c|c|c|c|c|}
\hline \multirow[b]{2}{*}{ Isoflavonoids } & \multicolumn{3}{|c|}{ Environmental factors } & \multicolumn{3}{|c|}{ Biotic factors (fungal elicitor) } \\
\hline & Control & Light & Dark & Control & P. sojae & Oligosaccharide \\
\hline \multirow[t]{2}{*}{ Daidzein } & 1.5 & 2.5 & 0.9 & 1.3 & 2.3 & 0.9 \\
\hline & \pm 0.1 & \pm 0.6 & \pm 0.2 & \pm 0.1 & \pm 0.7 & \pm 0.1 \\
\hline \multirow{2}{*}{ Genistein } & 64.2 & 88.7 & 55.5 & 59.0 & 73.1 & 58.1 \\
\hline & \pm 5.2 & \pm 5.5 & \pm 4.1 & \pm 4.5 & \pm 4.9 & \pm 5.7 \\
\hline \multirow[t]{2}{*}{ Genistin } & 30.0 & 38.6 & 25.1 & 26.6 & 28.0 & 26.3 \\
\hline & \pm 3.0 & \pm 3.6 & \pm 2.9 & \pm 1.9 & \pm 1.8 & \pm 1.3 \\
\hline Daidzin & 0.0 & 0.0 & 0.0 & 0.0 & 0.0 & 0.0 \\
\hline \multirow[t]{2}{*}{ Total } & 95.7 & 129.8 & 81.5 & 86.9 & 103.4 & 85.3 \\
\hline & \pm 8.3 & \pm 9.7 & \pm 7.2 & \pm 6.5 & \pm 7.4 & \pm 7.1 \\
\hline $\begin{array}{l}\text { Percent of } \\
\quad \text { control }(\%)\end{array}$ & 100 & 135 & 84 & 100 & 118 & 98 \\
\hline
\end{tabular}

aThree independent experiments, $n=3$; values are means \pm standard deviation (SD).

Table 3B. Soluble Protein Concentrations $\left(\mathrm{mg} \cdot \mathrm{kg}^{-1}\right.$ Dry weight biomass) ${ }^{\mathrm{a}}$ in Garbanzo Bean (Cicer arietinum)

\begin{tabular}{|c|c|c|c|c|c|}
\hline \multicolumn{3}{|c|}{ Environmental factors } & \multicolumn{3}{|c|}{ Biotic factors (fungal elicitor) } \\
\hline Control & Light & Dark & Control & P. sojae & Oligosaccharide \\
\hline $14,506.3$ & $23,660.0$ & $10,502.3$ & $11,330.1$ & $21,513.3$ & $12,587.9$ \\
\hline \pm 1005.3 & \pm 2125.2 & \pm 859.7 & \pm 968.6 & \pm 2500.5 & \pm 950.8 \\
\hline Control (\%) & $\begin{array}{l}\text { Percent of } \\
\text { control (\%) }\end{array}$ & $\begin{array}{l}\text { Percent of } \\
\text { control (\%) }\end{array}$ & Control $(\%)$ & $\begin{array}{l}\text { Percent of } \\
\quad \text { control }(\%)\end{array}$ & $\begin{array}{l}\text { Percent of } \\
\quad \text { control (\%) }\end{array}$ \\
\hline 100 & 163 & 72 & 100 & 190 & 111 \\
\hline
\end{tabular}

aThree independent experiments, $n=3$; values are means \pm standard deviation (SD). 
Table 4A. Isoflavonoid Concentrations $\left(\mathrm{mg} \cdot \mathrm{kg}^{-1}\right.$ DRy Weight BIOMAss) ${ }^{\mathrm{a}}$ in Fava BeAn (VICIA FABA)

\begin{tabular}{|c|c|c|c|c|c|c|}
\hline \multirow[b]{2}{*}{ Isoflavonoids } & \multicolumn{3}{|c|}{ Environmental factors } & \multicolumn{3}{|c|}{ Biotic factors (fungal elicitor) } \\
\hline & Control & Light & Dark & Control & P. sojae & Oligosaccharide \\
\hline \multirow[t]{2}{*}{ Daidzein } & 25.2 & 36.9 & 20.0 & 20.1 & 32.0 & 18.1 \\
\hline & \pm 2.0 & \pm 2.6 & \pm 1.5 & \pm 2.5 & \pm 2.6 & \pm 1.8 \\
\hline \multirow[t]{2}{*}{ Genistein } & 31.0 & 48.5 & 27.6 & 25.5 & 28.4 & 27.3 \\
\hline & \pm 2.0 & \pm 3.6 & \pm 3.5 & \pm 3.4 & \pm 2.8 & \pm 1.6 \\
\hline \multirow[t]{2}{*}{ Genistin } & 17.9 & 36.7 & 8.3 & 14.1 & 19.7 & 13.4 \\
\hline & \pm 1.1 & \pm 2.5 & \pm 0.6 & \pm 0.9 & \pm 1.1 & \pm 1.7 \\
\hline \multirow[t]{2}{*}{ Daidzin } & 14.6 & 22.1 & 7.0 & 11.1 & 13.3 & 9.0 \\
\hline & \pm 2.1 & \pm 1.5 & \pm 0.4 & \pm 0.9 & \pm 0.8 & \pm 0.9 \\
\hline \multirow[t]{2}{*}{ Total } & 88.7 & 144.2 & 62.9 & 70.8 & 103.4 & 67.8 \\
\hline & \pm 7.2 & \pm 10.2 & \pm 6.0 & \pm 7.7 & \pm 7.3 & \pm 6.0 \\
\hline $\begin{array}{l}\text { Percent of } \\
\text { control }(\%)\end{array}$ & 100 & 162 & 71 & 100 & 145 & 96 \\
\hline
\end{tabular}

aThree independent experiments, $n=3$; values are means \pm standard deviation (SD).

Table 4B. Soluble Protein Concentrations $\left(\mathrm{mg} \cdot \mathrm{kg}^{-1}\right.$ dry weight biomass)a in Fava Bean (Vicia faba)

\begin{tabular}{|c|c|c|c|c|c|}
\hline \multicolumn{3}{|c|}{ Environmental factors } & \multicolumn{3}{|c|}{ Biotic factors (fungal elicitor) } \\
\hline Control & Light & Dark & Control & P. sojae & Oligosaccharide \\
\hline $26,359.0$ & $35,692.1$ & $15,360.2$ & $22,145.3$ & $29,365.1$ & $24,366.0$ \\
\hline \pm 2600.2 & \pm 2561.3 & \pm 977.4 & \pm 1501.1 & \pm 2651.4 & \pm 1548.7 \\
\hline Control $(\%)$ & $\begin{array}{l}\text { Percent of } \\
\text { control (\%) }\end{array}$ & $\begin{array}{l}\text { Percent of } \\
\quad \text { control }(\%)\end{array}$ & Control (\%) & $\begin{array}{l}\text { Percent of } \\
\text { control }(\%)\end{array}$ & $\begin{array}{l}\text { Percent of } \\
\quad \text { control (\%) }\end{array}$ \\
\hline 100 & 135 & 58 & 100 & 133 & 110 \\
\hline
\end{tabular}

aThree independent experiments, $n=3$; values are means \pm standard deviation (SD).

Table 5A. Isoflavonoid Concentrations $\left(\mathrm{mg} \cdot \mathrm{kg}^{-1}\right.$ Dry Weight biomass) ${ }^{\mathrm{a}}$ in Mung Bean (Phaseolus aureus)

\begin{tabular}{|c|c|c|c|c|c|c|}
\hline \multirow[b]{2}{*}{ Isoflavonoids } & \multicolumn{3}{|c|}{ Environmental factors } & \multicolumn{3}{|c|}{ Biotic factors (fungal elicitor) } \\
\hline & Control & Light & Dark & Control & P. sojae & Oligosaccharide \\
\hline \multirow[t]{2}{*}{ Daidzein } & 36.2 & 58.2 & 21.6 & 30.0 & 39.0 & 34.1 \\
\hline & \pm 2.5 & \pm 4.5 & \pm 2.1 & \pm 2.3 & \pm 3.9 & \pm 2.5 \\
\hline \multirow[t]{2}{*}{ Genistein } & 45.5 & 66.3 & 40.3 & 38.6 & 51.0 & 42.5 \\
\hline & \pm 3.6 & \pm 5.5 & \pm 5.6 & \pm 2.5 & \pm 4.0 & \pm 6.6 \\
\hline \multirow[t]{2}{*}{ Genistin } & 25.6 & 36.9 & 21.9 & 23.7 & 26.7 & 24.0 \\
\hline & \pm 1.1 & \pm 3.4 & \pm 2.7 & \pm 1.9 & \pm 1.5 & \pm 5.1 \\
\hline \multirow[t]{2}{*}{ Daidzin } & 29.7 & 41.3 & 20.7 & 27.5 & 38.1 & 31.2 \\
\hline & \pm 2.3 & \pm 3.6 & \pm 1.1 & \pm 3.6 & \pm 3.6 & \pm 2.0 \\
\hline \multirow[t]{2}{*}{ Total } & 137.0 & 202.7 & 104.5 & 119.8 & 154.8 & 131.8 \\
\hline & \pm 9.5 & \pm 17.0 & \pm 11.5 & \pm 10.3 & \pm 13.0 & \pm 16.2 \\
\hline $\begin{array}{l}\text { Percent of } \\
\text { control }(\%)\end{array}$ & 100 & 148 & 77 & 100 & 129 & 110 \\
\hline
\end{tabular}

aThree independent experiments, $n=3$; values are means \pm standard deviation (SD).

Table 5B. Soluble Protein Concentrations $\left(\mathrm{mg} \cdot \mathrm{kg}^{-1}\right.$ dry weight biomass) ${ }^{\mathrm{a}}$ in Mung Bean (Phaseolus aureus

\begin{tabular}{|c|c|c|c|c|c|}
\hline \multicolumn{3}{|c|}{ Environmental factors } & \multicolumn{3}{|c|}{ Biotic factors (fungal elicitor) } \\
\hline Control & Light & Dark & Control & P. sojae & Oligosaccharide \\
\hline $20,014.3$ & $25,390.0$ & $14,522.1$ & $18,225.3$ & $23,618.3$ & $19,000.3$ \\
\hline \pm 2012.4 & \pm 1521.5 & \pm 895.7 & \pm 1052.3 & \pm 2500.6 & \pm 1689.8 \\
\hline Control (\%) & $\begin{array}{l}\text { Percent of } \\
\text { control }(\%)\end{array}$ & $\begin{array}{l}\text { Percent of } \\
\text { control }(\%)\end{array}$ & Control (\%) & $\begin{array}{l}\text { Percent of } \\
\text { control (\%) }\end{array}$ & $\begin{array}{l}\text { Percent of } \\
\text { control (\%) }\end{array}$ \\
\hline 100 & 127 & 73 & 100 & 130 & 104 \\
\hline
\end{tabular}

aThree independent experiments, $n=3$; values are means \pm standard deviation (SD). 
Table 6A. Isoflavonoid Concentrations $\left(\mathrm{mg} \cdot \mathrm{kg}^{-1}\right.$ Dry Weight biomass) ${ }^{\mathrm{a}}$ in Adzuki Bean (Phaseolus acutifolius)

\begin{tabular}{|c|c|c|c|c|c|c|}
\hline \multirow[b]{2}{*}{ Isoflavonoids } & \multicolumn{3}{|c|}{ Environmental factors } & \multicolumn{3}{|c|}{ Biotic factors (fungal elicitor) } \\
\hline & Control & Light & Dark & Control & P. sojae & Oligosaccharide \\
\hline \multirow[t]{2}{*}{ Daidzein } & 26.3 & 45.0 & 9.6 & 22.0 & 26.3 & 20.0 \\
\hline & \pm 2.5 & \pm 4.1 & \pm 1.0 & \pm 1.7 & \pm 2.4 & \pm 1.9 \\
\hline \multirow[t]{2}{*}{ Genistein } & 56.3 & 70.2 & 33.2 & 44.1 & 54.3 & 43.2 \\
\hline & \pm 4.2 & \pm 6.3 & \pm 2.3 & \pm 3.6 & \pm 3.6 & \pm 4.1 \\
\hline \multirow[t]{2}{*}{ Genistin } & 27.0 & 32.1 & 16.3 & 21.3 & 25.0 & 19.0 \\
\hline & \pm 1.6 & \pm 2.7 & \pm 1.3 & \pm 1.7 & \pm 1.9 & \pm 2.3 \\
\hline \multirow[t]{2}{*}{ Daidzin } & 16.6 & 20.0 & 0.2 & 10.0 & 12.0 & 9.9 \\
\hline & \pm 1.1 & \pm 1.5 & \pm 0.1 & \pm 0.9 & \pm 0.6 & \pm 0.8 \\
\hline \multirow[t]{2}{*}{ Total } & 126.2 & 167.1 & 59.3 & 97.4 & 117.6 & 92.1 \\
\hline & \pm 9.4 & \pm 14.6 & \pm 4.6 & \pm 7.9 & \pm 8.5 & \pm 9.1 \\
\hline $\begin{array}{l}\text { Percent of } \\
\text { control (\%) }\end{array}$ & 100 & 132 & 47 & 100 & 122 & 95 \\
\hline
\end{tabular}

aThree independent experiments, $n=3$; values are means \pm standard deviation (SD).

Table 6B. Soluble Protein Concentrations ( $\mathrm{mg} \cdot \mathrm{kg}^{-1}$ Dry weight biomass) a in Adzuki Bean (Phaseolus acutifolius)

\begin{tabular}{|c|c|c|c|c|c|}
\hline \multicolumn{3}{|c|}{ Environmental factors } & \multicolumn{3}{|c|}{ Biotic factors (fungal elicitor) } \\
\hline Control & Light & Dark & Control & P. sojae & Oligosaccharide \\
\hline $23,106.4$ & $35,390.3$ & $11,369.2$ & $20,000.3$ & $23,505.6$ & $18,506.2$ \\
\hline \pm 2333.3 & \pm 3500.2 & \pm 1501.1 & \pm 2085.3 & \pm 2310.4 & \pm 1204.7 \\
\hline Control (\%) & $\begin{array}{l}\text { Percent of } \\
\text { control }(\%)\end{array}$ & $\begin{array}{l}\text { Percent of } \\
\text { control }(\%)\end{array}$ & Control $(\%)$ & $\begin{array}{l}\text { Percent of } \\
\quad \text { control }(\%)\end{array}$ & $\begin{array}{l}\text { Percent of } \\
\text { control (\%) }\end{array}$ \\
\hline 100 & 153 & 50 & 100 & 118 & 93 \\
\hline
\end{tabular}

aThree independent experiments, $n=3$; values are means \pm standard deviation (SD).

plants that is responsible for the synthesis of secondary metabolites, such as isoflavonoids, and primary metabolites, such as soluble proteins (Stafford, 1990).

\section{DISCUSSION}

Our data and methods have demonstrated that upregulation of levels of isoflavonoids and soluble proteins in leguminous seedlings is not only possible, but also, easy to accomplish by simply germinating the legume seeds in the light rather than in the dark as is usually done. As an alternative to mung bean, soybean, fava bean, adzuki bean, or garbanzo bean sprouts, kudzu sprouts may offer a far better source of genistein and daidzein isoflavonoids (compare Tables 1-6) and soluble proteins. This may be of some importance to vegetarians, and certainly, as a complementary and alternative medicine modality. It is important to stress that roots be included in legume seedlings that are harvested because they contain the highest levels of isoflavonoids (Kaufman et al., 1997). Furthermore, these leguminous plants are intended to be eaten as sprouts, rather than as fully grown plants.

\section{CONCLUSIONS}

In the future, studies should be designed to test the effects of isoflavonoids derived from edible leguminous seedlings on patients in well-designed double-blinded clinical trials. Integrating isoflavonoid-based treatments into mainstream medicine could prove to be highly beneficial to patients, particularly because isoflavonoid therapy is relatively inexpensive compared to the currently prescribed cancer drugs, and there are few adverse side-effects.

In countries where proper medical care is not easily accessible, legume-based diets can be a great asset to a community. Our results have shown that light-elicited upregulation of iso- 
flavonoids and soluble proteins is easily accomplished and can significantly improve the protein and isoflavonoid contents of various edible legumes.

\section{ACKNOWLEDGEMENTS}

This research is based on a University of Michigan Department of Biology Senior Honor's thesis, completed in April 2002, by Ashish Lal. This study was supported in part by the University of Michigan National Institutes of Health National Center for Complementary and Alternative Medicine grant I P50 AT00011.

\section{REFERENCES}

Boik J. Cancer and Natural Medicine: A Textbook of Basic Science and Clinical Research. Princeton, MN: Oregon Medical Press, 1996.

Bradford MM. A rapid and sensitive technique for the quantitation of microgram quantities of protein utilizing the principle of protein-dye binding. Anal Biochem 1976;72:248-254.

Dixon R, Dey P, Lamb C. Phytoalexins: Enzymology and molecular biology. Adv Enzymol Relat Areas Mol Biol 1983;55:1-136.

Duke J. Commentary: Novel psychotherapeutic drugs. A role for ethnobotany. Psychopharmacol Bull 1995;31: 177-182.

Fotsis T, Pepper M, Aldercreutz HT, Montesano R., Schweiger L. Genistein, a dietary ingested isoflavonoid, inhibits cell proliferation and in vitro angiogenesis. J Nutr 1995;125:790S-797S.

Kaufman PB, Coon CW, Govil JN, Hoyt JE, Lu CR, Madsen BJ, Warber, SL. Creating a Sustainable Future, Living in Harmony with the Earth. New Delhi, India: Researchco Book Centre, 2002.

Kaufman PB, Duke JA, Brielmann H, Boik J, Hoyt JE. A comparative survey of leguminous plants as sources of the isoflavones, genistyein and daidzein: Implications for human nutrition and health. J Altern Complement Med 1997;3:7-12.

Keung WM, Vallee BL. Daidzin: A potent, selective inhibitor of human mitochondrial aldehyde dehydrogenase. Proc Nat Acad Sci 1993a;90:1247-1251.

Keung WM, Vallee BL. Daidzin and daidzein suppress free-choice ethanol intake in Syrian golden hamsters. Proc Nat Acad Sci 1993b;90:10008-10012.

Stafford H. Flavonoid Metabolism. Boca Raton, FL: CRC Press, 1990.

Address reprint requests to: Peter B. Kaufman, Ph.D. Department of Molecular, Cellular, and Developmental Biology University of Michigan Ann Arbor, MI 48109-1048

E-mail: pbk@umich.edu 
This article has been cited by:

1. Ara Kirakosyan, Peter B. Kaufman, Soo Chul Chang, Sara Warber, Steven Bolling, Hrachik Vardapetyan. 2006. Regulation of isoflavone production in hydroponically grown Pueraria montana (kudzu) by cork pieces, XAD-4, and methyl jasmonate. Plant Cell Reports 25:12, 1387-1391. [CrossRef] 